\title{
HIGHLIGHTS
}

PROSTATE CANCER

\section{Could the use of $5 \alpha$-reductase inhibitors during active surveillance reduce the risk of disease progression?}

5a-reductase inhibitors (5-ARIs) such as finasteride and dutasteride prevent the conversion of testosterone to dihydrotestosterone, a promoter of both benign and malignant growth of prostate tissue. Studies have indicated that this antiandrogenic effect might make this class of drug useful for preventing the occurrence-and, potentially, the progression-of prostate cancer. As active surveillance is becoming an increasingly popular management strategy for men with low-risk prostate cancer, Finelli et al. performed a retrospective, single-center study to examine whether concurrent 5-ARI use delayed pathologic progression in patients managed with this approach.

Overall, 288 men met the inclusion criteria for the analysis (serum PSA level $<10 \mathrm{ng} / \mathrm{ml}$, clinical stage T1c-T2a, Gleason score $<6, \leq 3$ positive cores and no cores with $>50 \%$ cancer involvement at the time of initial diagnostic biopsy). Patients were not included if they had used 5-ARIs before the diagnosis of prostate cancer. A confirmatory biopsy was performed 12 months after the initial biopsy, and every 2-3 years thereafter. Active surveillance included PSA measurement every 3-6 months and digital rectal examination every 6 months. Of the 288 men included, 70 were started on a 5-ARI before the diagnostic biopsy. The median follow-up duration was 38.5 months.

Pathologic progression (defined as increased tumor grade, $>3$ positive cores

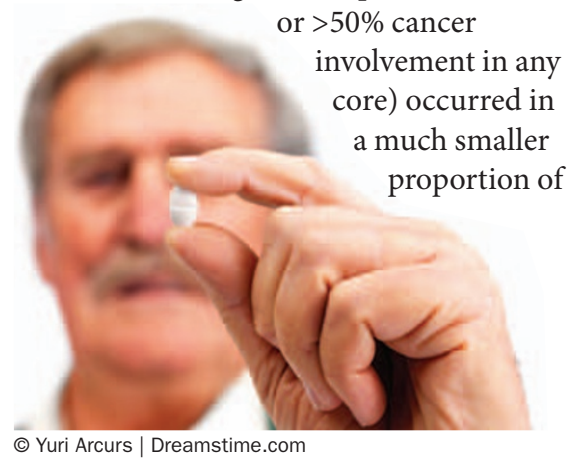

men in the 5-ARI group compared with the non-5-ARI group (18.6\% versus $36.7 \%$, $P=0.004)$, and the time to progression was significantly longer in men receiving a 5-ARI (41.3 months versus 35.1 months, $P=0.013)$. Multivariate analysis showed that lack of 5-ARI use was strongly associated with disease progression (hazard ratio 2.91, 95\% CI 1.5-5.6).

Despite the retrospective nature of the study, the authors conclude that the use of 5-ARIs during active surveillance seems to reduce the likelihood of progression. The forthcoming results of the large randomized, placebo-controlled REDEEM trial should shed more light on the effectiveness of 5-ARIs in this setting.

Nick Warde

Original article Finelli, A. et al. Impact of $5 a$-reductase inhibitors on men followed by active surveillance for prostate cancer. Eur. Urol. doi:10.1016/j.eururo.2010.12.018 